\title{
Pulmonary Vein
}

National Cancer Institute

\section{Source}

National Cancer Institute. Pulmonary Vein. NCI Thesaurus. Code C12776.

One of four veins that carries oxyg en-rich blood from the lungs to the cardiac left atrium. 